\title{
Rugosidade superficial de diferentes resinas compostas comparando sistemas de acabamento e polimento e após a profilaxia com jato de bicarbonato - Estudo in vitro
}

\author{
- Carol Brandt Alves Mestre em Dentistica Restauradora pela Universidade de São Paulo (USP) • Jéssika Barcellos \\ Giuriato Mestranda em Dentística Restauradora pela Universidade de São Paulo (USP) • Miriam Lacalle Turbino Professora \\ Doutora do Departamento de Dentistica da Universidade de São Paulo (USP) • Margareth Oda Professora Doutora do \\ Departamento de Dentistica da Universidade de São Paulo (USP)
}

RESUMO || A presente pesquisa in vitro teve como objetivo avaliar a superfície de três resinas compostas quando submetidas a tratamentos de polimento e jato de bicarbonato. As alterações foram avaliadas pela análise rugosimétrica. Foram testadas três resinas compostas, de diferentes tamanhos de partículas - nanohíbrida (Z350 XT- 3M ESPE), microhíbrida (Z250 - 3M ESPE) e microparticulada (Durafill - Heraeus Kulzer)-. Para cada resina foram confeccionados quinze corpos de prova aleatoriamente divididos em 3 grupos: polimento com pontas de silicone (Enhace+PoGo - Dentsply), pontas de borracha (astropol - Ivoclar Vivadent) e discos fléxiveis (Sof-Lex - 3M ESPE). A rugosidade das amostras foi analisada por meio do Rugosímetro (Mitutoyo SJ- 201P- Tókio- Japan). A mensuração da rugosidade foi realizada antes e após o polimento. Em seguida, cada grupo foi submetido à termociclagem e ao jato de bicarbonato, quando foi realizada a ultima leitura da rugosidade. A análise de variância mostrou haver diferença estatisticamente significante $(\mathrm{p}<0,05)$ entre as resinas ( $\mathrm{p}=0,0008)$, entre os polimentos $(\mathrm{p}=0,0001)$, e na interação entre resina e polimento ( $\mathrm{p}=0,007)$, porém entre os momentos não houve diferença estatisticamente significante $(\mathrm{p}=0,305)$. Dentre as resinas avaliadas a que apresentou maior grau de rugosidade foi a microparticulada (Durafill - Heraeus Kulzer), e o sistema de polimento que mostrou menor eficiência foi o sistema que utiliza pontas de borracha (Astropol - Ivoclar Vivadent).

DESCRITORES | Restauração Dentária Permanente; Polimento Dentário; Resinas Compostas.

ABSTRACT | This in vitro study aimed to evaluate the surface of three composite resins when subjected to polishing and bicarbonate jet treatments. Changes were evaluated by analysis rugosimetric (Ra). Three composite resins of different particle sizes were tested - Nanohybrid (XT-Z350 3M ESPE), microhybrid (Z250 - 3M ESPE) and microfilled (Durafill - Heraeus Kulzer). For every fifteen resin specimens were randomly divided into 3 groups: polishing with rubber tips (Astropol - Ivoclar Vivadent), floppy disks (Sof-Lex - 3M ESPE) and silicone tips (Dentsply Enhace + PoGo). The roughness of the samples was analyzed by the Surface Roughness Tester (Mitutoyo SJ-201P, Tokyo, Japan). The roughness measurement was performed before and after polishing. Afterward each group was subjected to thermocycling and bicarbonate jet, when the last roughness reading was performed. The analysis of variance showed a statistically significant difference between resins $(\mathrm{p}=0.0008)$, between polishing $(\mathrm{p}=0.0001)$, and interaction between resin and polishing $(\mathrm{p}=0.007)$ but between times there was no statistically significant difference $(\mathrm{p}=0.305)$. Among the resins evaluated,the highest roughness degree foundwas microfilled (Durafill - Heraeus Kulzer), while polishing system showed the lowest efficiency for rubber tips system (Astropol - Ivoclar Vivadent).

DESCRIPTORS | Dental Restoration Permanent; Dental Polishing; Composite Resins.

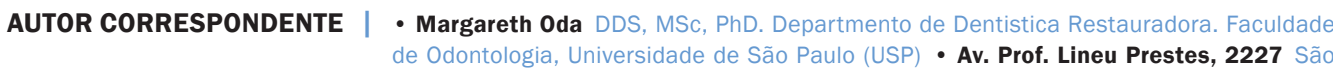
Paulo, SP, Brazil • 05508-900 E-mail: mege@usp.br

- Received Aug 04, 2015 • Accepted Feb 26, 2015

- Dol http://dx.doi.org/10.11606/issn.2357-8041.clrd.2015.83527 


\section{INTRODUÇÃO}

O surgimento das resinas compostas representa uma evolução na área da Odontologia Restauradora, pois permite a realização de restaurações com maior preservação da estrutura dentária, quando comparadas com outras técnicas restauradoras estéticas indiretas, além de proporcionar excelente estética. São também econômicas em relação ao tempo, pois não existe a fase laboratorial. ${ }^{1,2}$

O acabamento e o polimento das restaurações influenciam diretamente na durabilidade e na estética final. A presença de irregularidades promove a maior retenção de biofilme, descoloração da superfície, irritação gengival, aumento da solubilidade da matriz orgânica e a ocorrência de cárie secundaria. ${ }^{3}$ Segundo Antonson et al., ${ }^{4}$ fatores como tipo de resina composta, de monômero, concentração e tipo de carga e os sistemas de acabamento e polimento utilizados podem influenciar na lisura superfícial final da resina composta.

Para que uma restauração tenha aspecto semelhante ao de um dente hígido, como é exigido pela elevada demanda estética, é tecnicamente importante realizar o tratamento de acabamento e polimento de superfície para a restauração alcançar a textura adequada e apresentar um brilho natural, que permita a reflexão de luz. ${ }^{5} \mathrm{~A}$ característica superficial de uma restauração estética influi na sua durabilidade clínica. Devem-se reproduzir as propriedades da estrutura dentária referentes à cor, translucidez e textura, possibilitando acabamento e polimento adequados. ${ }^{6}$

Ao se restaurar com resina composta o principal objetivo do acabamento é a obtenção de uma restauração com contorno, formas de ameia, oclusão adequada e superfície regular. Além disso, a perfeita adaptação e vedamento marginal são importantes detalhes requeridos. Existem diferentes instrumentos para acabamento de restaurações de resina composta. No entanto, se não forem cuidadosamente utilizados, o acabamento pode levar à formação de fendas e comprometer a adaptação marginal.7,8

O ideal seria que após uma restauração a superfície se mostrasse o mais lisa e regular possível. Tal resultado, entretanto, nem sempre é possível. A presença de fatores como técnica de acabamento e polimento das restaurações, ${ }^{9,10}$ a ação mecânica da escovação dentária associada ao uso de dentifrícios, ${ }^{11}$ a ação abrasiva de jatos de ar/água/bicarbonato, ${ }^{12}$ bebidas, ${ }^{13,14}$ alimentos, ${ }^{15-17}$ aplicações tópicas de fluoretos, ${ }^{18}$ componentes salivares, alterações de $\mathrm{pH}$ de soluções orgânicas ${ }^{19-21} \mathrm{e}$ a ação de agentes clareadores, ${ }^{22}$ podem, quando associados em diferentes combinações, influenciar nas propriedades dos materiais restauradores, inclusive no maior ou menor grau de rugosidade superficial. ${ }^{22,23}$

Após o término de uma restauração duas etapas devem ser realizadas com a finalidade de se conseguir textura e lisura semelhante ao do dente. A etapa de acabamento elimina a camada superficial da restauração rica em matriz orgânica, e esta remoção expõe ou desloca as partículas inorgânicas, aumentando a rugosidade superficial. ${ }^{24} \mathrm{~A}$ etapa de acabamento pode aumentar em até 10 vezes a rugosidade superficial da resina, ao deixar marcas das lâminas presentes nas brocas utilizadas. A escolha do instrumento de acabamento é altamente significativa na lisura final de restaurações de resina composta. ${ }^{25,26} \mathrm{O}$ polimento ideal deve proporcionar um grau de lisura semelhante ao do esmalte, que tem valor médio de 0,28 $\mu \mathrm{m}$ de rugosidade. ${ }^{26}$

Segundo Watanabe et al., ${ }^{27}$ o valor clinicamente aceitável de rugosidade de restauração é de $0,2 \mu \mathrm{m}$. Endo et al., ${ }^{26}$ no entanto, afirmam que valores entre 0,25 e 0,5 $\mu \mathrm{m}$ de rugosidade já podem ser sentidos pelos pacientes com a ponta da língua.

A aplicação do jato de bicarbonato de sódio é uma técnica que apresenta excelentes resultados na remoção de manchas extrínsecas, como as causadas por alimentos, bebidas, nicotina e também do 
biofilme dental. ${ }^{28} \mathrm{O}$ seu uso, porém, pode causar aumento da rugosidade superficial da resina composta, fazendo com que perca as suas características em longo prazo e resulte em cáries secundárias, entre outros. ${ }^{8}$

Mediante as explanações sobre o assunto exposto, o objetivo dessa pesquisa foi avaliar e comparar, in vitro, a rugosidade superficial de três resinas compostas, de diferentes tamanhos de partículas, utilizando três sistemas de polimento, e também verificar a rugosidade após a profilaxia com jato de bicarbonato após serem submetidas a processo de termociclagem.

\section{MÉTODOS}

Foram utilizados três tipos de resinas compostas de diferentes tamanhos de partículas inorgânicas (Tabela 1):

- Microhíbrida (Filtek Z250 3M ESPE; Dental

Products, St Paul, MN, USA (FZ250)

- Microparticulada (Durafill VS Heraeus Kulzer

Weihrheim, Germany (DVS)

- Nanohíbrida (Filtek Z350 XT 3M ESPE; Dental

Products, St Paul, MN, USA)

Para cada resina foram confeccionados 15 corpos de prova. Sobre uma mesa plana, foi colocada uma lâmina de vidro sobre a qual foi colocada uma tira de poliéster, onde foi depositada a matriz de teflon de $7 \mathrm{~mm}$ de diâmetro e $3 \mathrm{~mm}$ de altura. A resina composta foi inserida em porção única nessa matriz e colocou-se sobre esta uma nova tira de poliéster e uma lâmina de vidro, a fim de padronizar a confecção dos corpos de prova. Cada resina foi fotoativada pelo tempo orientado pelo fabricante com fotoativador de luz LED Elipar Freelight (3M ESPE Dental Products, St Paul, MN, USA), com intensidade de $1.200 \mathrm{~mW} / \mathrm{cm}^{2}$. Após a polimerização, o corpo de prova foi removido da matriz e imediatamente imerso em água destilada a $37^{\circ} \mathrm{C}$, por $24 \mathrm{hs} .{ }^{21}$

Decorrido o período de 24horas, os corpos de prova foram retirados da água destilada, secos com jatos de ar e divididos aleatoriamente em 3 grupos: pontas de borracha (Astropol), pontas de silicone (Enhace $\AA+P o G o-2$ passos) e discos fléxiveis (SofLex) (3 passos) (Quadro 1).

Todos os grupos foram submetidos à mensuração da rugosidade por meio do rugosímetro (Mitutoyo SJ- 201P- Tókio- Japan), que possui uma ponta apalpadora com tamanho de $0,005 \mathrm{~mm}$ de raio que se desloca a uma velocidade de $0,5 \mathrm{~mm} / \mathrm{s}$, conectada a um visor de cristal líquido que registra digitalmente a leitura da superfície. O comprimento de amostragem foi de $0,25 \mathrm{~mm}$ e o percurso de medição de 1,25 mm. A calibragem prévia foi realizada sobre uma superfície padrão pertencente ao próprio equipamento.

Cada corpo de prova foi medido em cinco pontos diferentes. A primeira leitura foi realizada antes do

Tabela 1 | Especificações das resinas compostas utilizadas

\begin{tabular}{|c|c|c|c|c|c|c|}
\hline Material & Tipo/Cor & $\begin{array}{l}\text { Composição } \\
\text { Orgânica }\end{array}$ & Carga Inorgânica & $\begin{array}{l}\text { Porcentagem de } \\
\text { Particulas }\end{array}$ & Tamanho Médio & Fabricante \\
\hline Filtek z350 & $\mathrm{A} 2$ & $\begin{array}{l}\text { BisGma } \\
\text { UDMA } \\
\text { TEGDMA } \\
\text { Bis-EMA }\end{array}$ & Zircônia Sílica & $\begin{array}{c}\text { 72,5\% em Peso } \\
55,6 \% \text { em Volume }\end{array}$ & $\begin{array}{l}\text { Nanohíbrida } \\
\text { (0,6 a 1,4nm) }\end{array}$ & 3m Espe \\
\hline Filtek z250 & A2 & $\begin{array}{l}\text { BisGma } \\
\text { UDMA } \\
\text { Bis-EMA }\end{array}$ & Zircônia Sílica & $60 \%$ em Volume & $\begin{array}{c}\text { Microhíbrida } \\
(0,01 \mu \mathrm{m} \text { a } 3,5 \\
\mu \mathrm{m}\end{array}$ & 3M Espe \\
\hline Durafill vs & $\mathrm{A} 2$ & $\begin{array}{c}\text { BisGma } \\
\text { TEGDMA } \\
\text { DIMETACRILATO } \\
\text { de Uretano }\end{array}$ & Dióxido de Silício & $66 \% \mathrm{v} / \mathrm{v}$ & 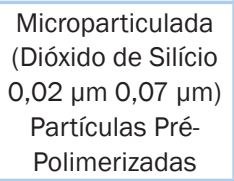 & Heraeus \\
\hline
\end{tabular}


Quadro 1 | Tamanho das partículas dos materiais para acabamento

\begin{tabular}{c|c|c|c|c} 
& Grosso & Médio & Fino & Superfino \\
\hline \begin{tabular}{c|c} 
Soflex \\
$\begin{array}{c}\text { Astropol } \\
\text { (Ivoclair) }\end{array}$
\end{tabular} & $55 \mu \mathrm{m}$ & $40 \mu \mathrm{m}$ & $24 \mu \mathrm{m}$ & $8 \mu \mathrm{m}$ \\
\hline Enhance (Caulk) & & $40 \mu \mathrm{m}$ & $20-40 \mu \mathrm{m}$ & $10 \mu \mathrm{m}$ \\
\hline
\end{tabular}

polimento (controle). Após esse passo todos os corpos de prova receberam subsequente acabamento superficial, onde foi utilizada uma ponta diamantada 3168 F (KG Sorensen), seguida da ponta diamantada extrafina 3168FF (KG Sorensen), por um período de 15 segundos, em alta rotação e sob refrigeração com água.

Em seguida, cada grupo recebeu polimento utilizando-se os sistemas descritos anteriormente, sempre seguindo as orientações do fabricante. $\mathrm{Na}$ sequência de cada técnica os corpos de prova foram lavados em água da seringa tríplice após cada ponta ou disco.

Após o polimento, os corpos de prova foram submetidos ao sistema de envelhecimento acelerado, termociclagem com 1000 ciclos e variação de temperatura de $5^{\circ} \mathrm{C}$ a $55^{\circ} \mathrm{C}$ (Ciclagem térmica série 521-4D.Nova Ética, Brasil).

Após a termociclagem foi feita nova análise de rugosidade superficial. Em seguida os corpos de prova foram submetidos ao jato de bicarbonato de sódio durante 30 segundos, a uma distância de aproximadamente $1 \mathrm{~cm}$ da superfície do corpo de prova com o equipamento de profilaxia Bio Jato Ortus (Ortus Ind. E Com. LTDA). Após esse tratamento foi efetuada uma nova mensuração da rugosidade superficial.

\section{RESULTADOS}

Foram obtidos 405 valores de Ra correspondentes a 3 resinas, 3 técnicas de polimento, 3 momentos, 5 repetições e 3 mensurações ( 3 × 3 × 3 × 5 × 3=405).

Para a análise estatística foram calculadas as médias das 3 mensurações em cada corpo de prova. Os 405 valores foram submetidos a testes de normalidade e homogeneidade, e demonstraram tratar-se de distribuição normal e homogênea.

As médias de todos os grupos analisados são apresentadas na tabela 2. Para a comparação entre as médias foi calculado o valor Tukey para interação resina x polimento x momento $(\mathrm{T}=0,48)$.

Pode-se notar que a resina microparticulada foi a que apresentou os maiores valores de Ra, principalmente após o jato de bicarbonato.

Tabela 2 | Valores da média da rugosidade superficial (parâmetro Ra)

\begin{tabular}{|c|c|c|c|c|c|c|c|c|c|}
\hline & \multicolumn{3}{|c|}{ Controle } & \multicolumn{3}{|c|}{ Após Polimento } & \multicolumn{3}{|c|}{ Após Jato de Biocarbonato } \\
\hline & Z350 & $\mathrm{Z} 250$ & Durafill & Z350 & $\mathrm{Z} 250$ & Durafill & $\mathrm{Z} 350$ & $\mathrm{Z} 250$ & Durafill \\
\hline Enhance+PoGo & $\begin{array}{c}\text { A } \\
0,46 \\
a\end{array}$ & $\begin{array}{c}\text { A } \\
0,39 \\
a\end{array}$ & $\begin{array}{c}\text { A } \\
0,5 \\
a\end{array}$ & $\begin{array}{c}A B \\
0,34 \\
a\end{array}$ & $\begin{array}{c}\text { A } \\
0,34 \\
a\end{array}$ & $\begin{array}{c}\text { A } \\
0,41 \\
a\end{array}$ & $\begin{array}{c}A B \\
0,29 \\
a\end{array}$ & $\begin{array}{c}\text { A } \\
0,36 \\
a\end{array}$ & $\begin{array}{c}\text { A } \\
028 \\
a\end{array}$ \\
\hline Astropol & $\begin{array}{c}\text { A } \\
0,51 \\
a\end{array}$ & $\begin{array}{c}\text { A } \\
0,41 \\
a\end{array}$ & $\begin{array}{c}\text { A } \\
0,45 \\
a\end{array}$ & $\begin{array}{c}B \\
0,73 \\
a\end{array}$ & $\begin{array}{c}\text { A } \\
0,49 \\
a\end{array}$ & $\begin{array}{c}\text { A } \\
0,77 \\
a\end{array}$ & $\begin{array}{c}B \\
0,72 \\
a\end{array}$ & $\begin{array}{c}\text { A } \\
0,41 \\
a\end{array}$ & $\begin{array}{c}B \\
1,14 \\
b\end{array}$ \\
\hline Sof-Lex & $\begin{array}{c}\text { A } \\
0,48 \\
a b\end{array}$ & $\begin{array}{c}\text { A } \\
0,33 \\
a b\end{array}$ & $\begin{array}{c}A \\
0,65 \\
b\end{array}$ & $\begin{array}{c}A \\
0,11 \\
a\end{array}$ & $\begin{array}{c}\text { A } \\
0,17 \\
\text { ab }\end{array}$ & $\begin{array}{c}A \\
0,43 \\
a b\end{array}$ & $\begin{array}{c}\text { A } \\
0,21 \\
a b\end{array}$ & $\begin{array}{c}\text { A } \\
0,29 \\
a b\end{array}$ & $\begin{array}{c}A \\
0,65 \\
b\end{array}$ \\
\hline
\end{tabular}

Médias de todos os grupos avaliados . Para a comparação entre essas médias foi calculado o valor Tukey para interação resina $X$ polimento $X$ momento $(\mathrm{T}=0,48)$.

OBS: letras diferentes representam grupos diferentes entre si na horizontal ,minúsculas, e na vertical, maiúsculas. 
Para a comparação entre os grupos foi calculada a ANOVA para 3 critérios, sendo dois deles independentes (resina e técnica de polimento) e um vinculado (momento de análise).

A ANOVA mostrou haver diferença estatisticamente significante entre as resinas $(p=0,0008)$, entre os polimentos ( $\mathrm{p}=0,0001)$ e na interação resina versus polimento $(\mathrm{p}=0,0074)$. Para o fator momento não houve diferença estatística $(\mathrm{p}=0,305)$.

Para o fator resina, o valor do Tukey $(\mathrm{T}=0.085)$ demonstrou não haver diferença estatística entre as resinas nanohíbrida $(\mathrm{m}=0,42)$ e microhíbrida $(m=0,35)$. A resina microparticualda $(m=0,58)$ apresentou maior rugosidade quando comparada às resinas micro e nanohíbridas.

Para o fator polimento, o valor do Tukey $(\mathrm{T}=0,085)$ mostrou que a rugosidade foi maior com o polimento utilizando pontas de borracha Astropol $(\mathrm{m}=0,62)$, sendo que os polimentos com discos flexíveis Sof-Lex $(\mathrm{m}=0,37)$ e pontas de silicone Enhance + PoGo $(m=0,36)$ foram menores e não apresentava diferença entre si.

As médias de todos os grupos avaliados são apresentadas na tabela 2 e as letras diferentes correspondem às médias estatisticamente distintas.

Para a análise estatística foram calculadas as médias de 3 mensurações em cada corpo de prova. Os 45 valores foram submetidos a testes de normalidade e homogeneidade, demonstrando tratar-se de uma amostra normal e homogênea.

\section{DISCUSSÃO}

Os materiais restauradores devem apresentar-se lisos, por isso procedimentos de acabamento e polimento são necessários após a confecção de uma restauração de resina composta, uma vez que minimizam a retenção do biofilme, manchas e outros resultantes da exposição de superfícies ásperas ao ambiente bucal, preservando a estética. ${ }^{3}$ Concordando com este procedimento, Dennison ${ }^{6}$ também concluiu em sua pesquisa que uma superfície altamente polida é clinicamente semelhante ao esmalte adjacente e deve ter um mínimo de retenção de biofilme, cuja dispersão da luz realce as características de textura do elemento dental.

Quando a resina é sujeita a abrasão, suas partículas são perdidas, gerando a rugosidade e consequentemente causando perda do potencial de refletividade da superfície da resina composta, ou seja, perda do polimento.

As resinas nanohíbridas, microhíbridas e microparticuladas contêm uma ampla quantidade de partículas, o que leva a um aumento da resistência do material e da resistência ao desgaste, o que pode explicar o fato do jato de bicarbonato não deixar a resina composta rugosa, já que não foi observada diferença entre os momentos de leitura, ou seja, controle, após polimento e após jato de bicarbonato.

Como as resinas nanohíbridas e microhíbridas contêm partículas de diferentes tamanhos em sua composição, quando submetidas ao polimento comportam-se melhor do que as resinas microparticuladas, que por terem partículas do mesmo tamanho acabam por perder algumas delas, ocasionando a formação de microcrateras, que são detectadas pelo rugosímetro.

O rugosímetro rastreia suavemente a superfície, registrando rugosidades. A superfície final da resina após o polimento deve ter uma aparência acetinada e brilhante. O processo de acabamento e de polimento é influenciado pela composição do substrato, tamanho de partícula do abrasivo, pressão aplicada, e tempo de aplicação. Técnicas sequenciais são normalmente utilizadas para melhorar a eficiência na obtenção da superfície o mais lisa possível. ${ }^{9}$ Com este entendimento justifica-se a utilização, nesta pesquisa, do cuidadoso acabamento e polimento com a técnica sequencial.

A literatura demonstra que a lisura obtida com a tira de poliéster é a melhor, ${ }^{3}$ ou seja, a rugosidade é menor; tal resultado, porém, não foi observado neste trabalho. Isso pode ser explicado pela 
provável formação de microbolhas na resina quando pressionada contra a tira de poliéster durante a polimerização, porém essas são removidas após o polimento. O polimento e o jato de bicarbonato não foram prejudiciais à lisura dos materiais e técnicas testadas.

Em geral, três etapas diferentes constituem as restaurações: contorno, acabamento e polimento. Usualmente, as pontas multilaminadas e diamantadas rotatórias além dos sistemas de discos de lixa de papel são utilizados para a redução de volume e de contorno. O acabamento remove os arranhões criados pelos instrumentos de contorno e proporciona uma superfície lisa. Durante o processo de acabamento, a restauração é contornada para combinar com a forma do dente e para estabelecer integridade marginal com a interface dente-restauração.7.7 $\mathrm{O}$ polimento é o processo final, o qual fornece brilho de esmalte à restauração, bem como reduz a energia de superfície da restauração. No processo de polimento, independentemente dos instrumentos utilizados, são usados discos e pontas rotatórias de níveis de granulação grossa a fina para remover irregularidades do material. Quando diferentes tipos de polimentos são propostos, devem ser considerados a manutenção de uma superfície lisa e também a manutenção do brilho da restauração. A medição do brilho é um parâmetro adicional de rugosidade para avaliar a eficácia do polimento. ${ }^{4,8,9}$

Alguns estudos ${ }^{3,22,24}$ reportam diferenças significativas entre os sistemas de polimento de discos flexíveis (Sof-Lex) e pontas de silicone. Nesta pesquisa, entretanto, observou-se quanto a todas as resinas avaliadas que o uso da sequência completa de discos flexíveis (Sof-Lex Pop On) e das pontas de silicone (Enhance+ PoGo) resultou em superfícies mais lisas e sem diferença estatística entre eles. Também foi observado que o sistema com pontas de borracha (Astropol) produziu os piores resultados para todas as resinas avaliadas com diferença significativa estatisticamente para os outros dois sistemas.

O polimento com discos flexíveis produz uma superfície mais lisa por não deslocar as cargas compostas. ${ }^{15,22}$ Os agentes de carga dos compósitos nanoparticulados são tão pequenos que sua rigidez é reduzida e, portanto, os discos flexíveis (Sof Lex Pop On) são recomendados porque a sua maleabilidade promove uma abrasão homogênea das cargas e da matriz, ${ }^{3}$ o que justifica os resultados de lisura adequada obtidos neste trabalho com o polimento dos discos flexíveis (Sof Lex) em todas as resinas utilizadas.

A profilaxia dental é uma prática rotineira para a remoção de biofilme e pode ser realizada de diversas maneiras. Escolhemos o Jato de Bicarbonato por ser um meio bem eficiente e prático, o que faz com que os Cirurgiões-Dentistas adiram esta técnica. Entretanto, como efeito secundário à limpeza, o Jato de Bicarbonato, por ser um sistema que libera jato de ar controlado, água e partículas de bicarbonato de sódio, pode causar uma superfície mais rugosa das resinas e favorecer o manchamento e degradações da restauração. ${ }^{22-24}$ Tal alteração, porém, não foi significante neste estudo, fato também observado no trabalho de Salami e Luz. ${ }^{9}$

\section{CONCLUSÃO}

De acordo com a metodologia empregada, pode-se concluir que os discos flexíveis (Sof-Lex Pop On) e o sistema de polimento com pontas siliconadas (Enhance + PoGo ) produziram superfícies mais lisas do que o sistema de polimento com as pontas de borracha (Astropol).

Dentre as resinas compostas a que apresentou maior rugosidade superficial foi a microparticulada (Durafill), enquanto as resinas nanohíbridas (Z350 XT) e microhíbrida (Z250) apresentaram a menor rugosidade. $\mathrm{O}$ jato de bicarbonato não alterou a rugosidade de superfície. 


\section{APLICAÇÃO CLÍNICA}

Na clínica odontológica procedimentos como a profilaxia com jato de bicarbonato são técnicas comuns e frequentemente utilizadas. Através deste trabalho comprovamos que este procedimento não prejudica a resina composta, que é o material restaurador estético mais utilizado.

Ao se realizar a etapa de polimento de uma restauração espera-se obter uma superfície regular, onde não se acumulem bactérias e pigmentos, que acabam por diminuir a vida útil da restauração. Os materiais que se mostraram mais eficaz neste quesito foram as pontas siliconadas e os discos flexíveis.

Para restaurações em dentes anteriores, as resinas que apresentaram as características mais adequadas foram as nanohíbridas e micro-híbridas, que devem ser as escolhidas quando a qualidade estética é requerida por obterem melhor polimento.

\section{REFERÊNCIAS}

1. Anfe TE, Caneppele TM, Agra CM, Vieira GF. Microhardness assessment of different commercial brands of resin composites with different degrees of translucence. Braz Oral Res. 2008 Oct-Dec;22(4):358-63. doi: 10.1590/S18o683242008000400013 .

2. Arhun N, Celik C, Yamanel K. Clinical evaluation of resinbased composites in posterior restorations: two-year results. Oper Dent. 2010 Jul-Aug;35(4):397-404. doi: 10.2341/o9345-C.

3. Scheibe KG, Almeida KG, Medeiros IS, Costa JF, Alves CM. Effect of different polishing systems on the surface roughness of microhybrid composites. J Appl Oral Sci. 2009 JanFeb;17(1):21-6. doi: 10.1590/S1678-77572009000100005.

4. Antonson SA, Yazici AR, Kilinc E, Antonson DE, Hardigan PC. Comparison of different finishing/polishing systems on surface roughness and gloss of resin composites. J Dent. 2011 Jul;39 Suppl 1:e9-17. doi: 10.1016/j. jdent.2011.01.006.

5. Marghalani HY. Effect of finishing/polishing systems on the surface roughness of novel posterior composites. J Esthet Restor Dent. 2010 Apr;22(2):127-38. doi: 10.1111/j.17088240.2010.00324.x.
6. Maresca C, Pimenta L, Heymann HO, Ziemiecki TL, Ritter AV. Effect of finishing instrumentation on the margin integrity of resin-based composite restorations. J Esthet Restor Dent. 2010 Apr;22(2):104-13. doi: 10.1111/j.17088240.2010.00320.x.

7. Dennison J. Commentary. Surface roughness and staining susceptibility of composite resins after finishing and polishing. J Esthet Restor Dent. 2011 Feb;23(1):44-5. doi: 10.1111/j.1708W-8240.2010.00377.x.

8. Costa JB, Gonçalves F, Ferracane JL. Comparison of twostep versus four-step composite finishing/polishing disc systems: evaluation of a new two-step composite polishing disc system. Oper Dent. 2011 Mar-apr;36(2):205-12. doi: 10.2341/10-162-L.

9. Salami D, Luz MAAC. Effect of prophylactic tratments on the superficial roughness of dental tissues and of two esthetic restorative materials. Pesqui Odontol Bras. 2003 jan.-mar.;17(1):63-8. doi: 10.159o/S151774912003000100012.

10. Ward DH. Esthetic restoration of tooth structure using a nonofill composite system. Compendium Contin Educ Dent. 2005 Apr;26(4):252, 254, 256-7.

11. Marquis JA, Murchison DF, Charlton DG, Cooley RL. Effect of air-powder abrasion prophylaxis on compomer surface roughness. J Dent Res. 2000;79(Abstract 1080):278.

12. Wimonchit S, Sarinnapakorn L. Effect of acidulated phosphate fluoride on surface roughness of composite resin. J Dent Res. 2000;79(Abstract 1074):278.

13. Yap AU, Low JS, Ong LF. Effect of food - simulating liquids on surface characteristics of composite and polyacid - modified composite restoratives. Oper Dent. 2000 MayJun;25(3):170-6. doi: 10.2341/11-419-L

14. Yap AU, Tan SH, Wee SS, Lee CW, Lim EL, Zeng KY. Chemical degradation of composite restoratives. J Oral Rehabil. 2001 Nov;28(11):1015-21. doi: 10.1111/j.1365-2842.2001.0076o.x

15. Kilimitzoglou D, Wolff MS. The surface roughness of a microfilm and hybrid composite after exposure to carbamide peroxide. J Dent Res. 2000;79(Abstract 1070):277.

16. Scougall-Vilchis RJ, Hotta Y, Hotta M, Idono T, Yamamoto K. Examination of composite resins with electron microscopy, microhardness tester and energy dispersive X-ray microanalyzer. Dent Mater J. 2009 Jan;28(1):102-12. doi: 10.4012/ dmj.28.102.

17. Barbosa SH, Zanata RL, Navarro MFL, Nunes OB. Effect of different finishing and polishing tecniques on the surface roughness of microfilled, hybrid and packable composite 
resins. Bras Dent J. 2005 jan.-abr.;16(1):39-44. doi: 10.1590/ S0103-64402005000100007.

18. Samra APB, Kossatz S, Borges CF, Ribeiro DG. Influence of professional prophylaxis on reducing discolouration of different aesthetic restorative materials. J Dent. 2012 Dec;40 Suppl 2:e71-6. doi: 10.1016/j.jdent.2012.06.003.

19. Pontes AP, Mainieri ET, Pacheco JF, Martins JL, Shinkai RAS, Mainieri VC. Rugosidade superficial de compósitos microparticulados e nanoparticulados após acabamento e polimento. Rev Gaúcha Odontol. 2009 abr.-jun.;57(2):179-82.

20. Hanadi Y, Marghaalani MS. Effect of Finishing/Polishing Systems on the Surface Roughness of Novel Posterior Composites. J Comp. 2010 Apr;22(2):127-38. doi: 10.1111/j.17088240.2010.00324.x.

21. Korkmaz Y, Ozel E, Attar N, Akasoy G. The influence of on step polishing systems on the surface roughness and microhardness and microhardness of nanocomposites. Operative dentistry. 2008. 33(1):44-50. doi: 10.2341/07-28.

22. Celik C, Arhun N, Yamanel K. Clinical evaluation of resinbased composites in posterior restorations: 12-month results. Eur J Dent. 2010 Jan;4(1):57-65.

23. Ikeda M, Matin K, Nikaido T, Foxton RM, Tagami J. Effect of surface characteristics on adherence of S. mutans biofilms to indirect resin composites. Dent Mater J. 2007 Nov;26(6): 915-23. doi: 10.4012/dmj.26.915.

24. Pereira CA, Eskelson E, Cavalli V, Liporoni PCS, Jorge AOC, Rego MA. Streptococcus mutans biofilm adhesion on composite resin surfaces after different finishing and polishing techniques. Oper Dent. 2011 May-Jun;36(3):311-7. doi: 10.2341/10-285-L.

25. Botta AC, Duarte Jr S, Paulin Filho PI, Gheno SM.Effect of dental finishing instruments on the surface roughness of composite resins as elucidated by atomic force microscopy. Microsc Microanal. 2008 Oct;14(5):380-6. doi: 10.1017/ S1431927608080768.

26. Endo T, Finger WJ, Kanehira M, Utterodt A, Komatsu M. Surface texture and roughness of polished nanofill and nanohybrid resin composites. Dent Mater J. 2010 Mar;29(2):21323. doi: 10.4012/dmj.2009-019.

27. Watanabe T, Miyazaki M, Moore BK. Influence of polishing instruments on the surface texture of resin composites. Quintessence Int. 2006 Jan;37(1):61-7.

28. Jones CS, Billington RW, Pearson GJ. The in vivo perception of roughnes of restorations. Br Dent J. 2004 Jan;196(1):42-5. doi: $10.1038 /$ sj.bdj.4810881. 